\section{Future applications of science with synchrotron radiation and free-electron lasers in Europe}

\author{
Guy Le Lay, ${ }^{a}$ Peter Lindley ${ }^{b}$ and Giorgio \\ Margaritondo $^{c_{*}}$
}

${ }^{a}$ CRMC2-CNRS, Campus de Luminy, Case 913, F-13288 Marseille CEDEX 9, France, ${ }^{b}$ European Synchrotron Radiation Facility, BP 220, F-38043 Grenoble, France, and ${ }^{C}$ Département de Physique, Ecole Polytechnique Fédérale de Lausanne, CH-1015 Lausanne, Switzerland.E-mail: marga@dpmail.epfl.ch

On 16-17 March 2001, many scientists from synchrotron and freeelectron laser (FEL) facilities met at the ESRF site in Grenoble for a workshop on 'Future Applications of Science with Synchrotron Radiation and Free-Electron Lasers in Europe'. The workshop was organized following several suggestions that emerged during the 2000 meeting of the European Round Table (RT) for Synchrotron Radiation and Free Electron Lasers in Karlsruhe. The main purpose was an open and broad discussion on the development of this field in Europe over the next 10-20 years. The objectives were a clarification of the possible development lines after the recent approval of the Soleil and Diamond projects, and, if possible, a consensus on priorities.

The participants included experts on accelerators and X-ray sources from Europe and the USA, and a limited number of X-ray users. A school on the same theme at Les Houches preceded the workshop in Grenoble. Two days of presentations and discussions were extremely helpful in clarifying the foreseeable technical development avenues. They also revealed several important points of broad consensus that could provide the basis for a realistic and effective long-term strategy in Europe.

The most relevant facts emerging from the workshops were the following:

(i) Synchrotron radiation and FEL facilities have been exceedingly successful in the previous 15 years, both in Europe and worldwide:

(a) The development of many major facilities did not encounter any failure;

(b) On the contrary, all projects significantly exceeded the promised performances with no additional cost;

(c) The number of users is still rapidly growing; all facilities are largely oversubscribed, in many cases to unacceptable levels;

$(d)$ The users spectrum is much broader and more diversified than 15 years ago, with a strong and rapidly expanding life-science component;

(e) The domain has produced its first Nobel prize and many other top-level awards.

Although the symposium was focused on X-ray and UV sources, one should note that FEL facilities operating in the IR are extremely successful and serve a large and active community of users. This remark applies, in particular, to the CLIO and FELIX facilities in Europe.

(ii) The present leading source technology based on storage rings has not yet reached its limits. Substantial improvements are still possible as far as stability, brightness, flux, coherence and polarization are concerned.

(iii) Novel technologies are emerging thanks to intense $\mathrm{R} \& \mathrm{D}$, primarily in Europe and the USA. All of them are based on LINACs rather than on storage rings. LINAC-based X-ray emission techniques can be divided into two broad subsets:

(a) Energy-recover LINACs (ERLs). This has been defined as an 'evolutionary' technology, which could potentially extend the performances of storage-ring-based sources.

(b) X-ray free-electron lasers (X-FELs) based on the SASE (selfamplified spontaneous emission) mechanism. This is a 'revolutionary' rather than evolutionary technology, whose main objective is to produce ultrashort (100-200 fs) X-ray pulses with extremely high peak brightness and flux and very high coherence.

The R\&D for ERLs is primarily centred in the USA, with an important contribution from Russia. The R\&D for X-FELs is shared between Europe (primarily HASYLAB in Germany) and the USA (primarily Argonne and Stanford).

The workshop stressed, in general, the scientific relevance of the new X-ray production technologies:

(i) The scientific case for pushing the storage-ring technology to the limit is well established, touching diverse domains of physics, chemistry, materials science, biology and other areas.

(ii) Similar considerations provide a strong scientific case for ERLs, that would constitute to some extent a continuation of the storage-ring technology.

(iii) A strong scientific case is also emerging for X-FELs, primarily based on the concentration of unprecedented X-ray flux into very short pulses. The X-FELs applications are largely complementary to those of storage-ring sources. These devices could broaden the use of $\mathrm{X}$-rays in directions not touched by storage rings, but could not replace storage rings.

The future evolution of the domain must deal with several important technical issues:

(i) The storage-ring technology is very mature, therefore its extension to the limit can be considered quite safe. However, significant performance improvements will still require a substantial amount of R\&D.

(ii) The ERL and X-FEL technologies are still under development. Important milestones were recently achieved by the TESLA project at HASYLAB and by Argonne. However, the full extension of the SASE technology to X-ray wavelengths requires the solution of important technical issues, concerning in particular the electron gun.

(iii) On the other hand, the aforementioned tests on SASE at HASYLAB and Argonne did not reveal any fundamental flaw in this emission mechanism.

The core discussion of the workshop was dedicated to the new programs in this domain that are emerging in Europe, notably initiatives that are being developed in Europe for novel X-ray sources:

(i) The practical concept of a 'limit' storage-ring facility for hard $\mathrm{X}$-rays is being developed within the ESRF.

(ii) HASYLAB has constructed and operated the TESLA test facility for the SASE mechanism, and has proposed an X-FEL in connection with a long linear collider for high-energy physics.

(iii) Italy has provided R\&D funding to develop a SASE X-FEL project.

(iv) The UK is considering a SASE FEL, limited to low photon energies $(<10 \mathrm{eV})$, within the CASIM project at Daresbury.

(v) Bessy in Germany is studying a low-photon-energy SASE FEL.

In addition, the development of a European strategy for the future of this domain requires several other important points to be taken into account:

(i) The $\mathrm{R} \& \mathrm{D}$ for future $\mathrm{X}$-ray production and use cannot be limited to new source technologies. Substantial parallel efforts are required for detectors, robotics and other areas. 
(ii) The increasing relative role of hard X-rays in synchrotron research should not lead to the extreme polarization of the domain. Substantial and targeted efforts are also required for UV and soft $\mathrm{X}$-ray research.

(iii) Again concerning the production and use of UV light and soft X-rays, impressive results were recently obtained by FELs based on storage rings (LURE, Elettra). Unfortunately, their operation interferes with the 'normal' use of the host storage rings. The possibility has been raised of a dedicated storage ring in Europe supporting several UV FELs.

(iv) Transnational access support has been a major factor in the success and expansion of synchrotron-based research in Europe. Programs supported by the EC and coordinated by the European Round Table, together with the user-support policy of the ESRF, practically opened the network of synchrotron and FEL facilities in Europe to all qualified European users.

(v) The shortage of qualified accelerator experts is becoming a major problem. Without effective measures, this is likely to become a critical bottleneck for all new initiatives.

The highlight of the workshop was the identification of the emerging points of consensus for the future of synchrotron light and FELs in Europe.

The open discussions during the workshop were extremely helpful in clarifying the priority issue. In the end, a broad consensus emerged on several key points:

(i) The need of national and European funding programs to guarantee the full instrumentation of the operating facilities, as well as of the facilities under development.

(ii) The need to continue and expand the transnational access support programs both through the ESRF and through EC contracts with 'national' facilities.

(iii) Full support for the rapid completion and commissioning of Soleil and Diamond.

(iv) Support for the project under development of an X-ray source at the limit of the storage-ring technology.

(v) Support for advanced R\&D on SASE FELs, followed by decisions on the development of European-level X-FEL facilities.

(vi) Support for the user operation of new advanced facilities in Europe in the UV and soft X-ray ranges: Tesla I, the Bessy
SASE FEL, CASIM, possibly a centralized storage-ring-based FEL facility.

Last, but not least, the workshop discussed the global financial issues underlying the strategic development of this domain in Europe: over the past 15 years, Europe invested reasonable resources into the development of an advanced network of synchrotron facilities, through both national and multi-country funding channels. The expanded user population, the broadened scope and the increasing industrial impact of synchrotron-based activities would certainly justify an increase in the investment rate for the next 15 years.

On the other hand, such an increase may not be necessary. The technology of X-ray sources has become exceedingly advanced, but it is not more expensive than in the past. The consequences are quite positive.

Future investments per year at a level similar to the past 15 years (corrected for inflation) would enable Europe to stay at the forefront of this domain. It appears specifically sufficient for the development of all the projects, which may emerge from the above consensus points. In other words, Europe can provide state-of-theart facilities for a much wider and more diversified scientific community without increasing its financial commitments with respect to the recent past.

After two days of intense discussion, several important conclusions emerged that could form the basis of a consensus strategy for the future of synchrotron light and FELs in Europe. The scientific cases, the technical issues and the financial projections clearly indicate that Europe should maintain all options open for the future development of its X-ray network. Through projects both at the national and panEuropean level, it should work along four parallel and complementary directions:

(i) Fully equipping the present facilities and rapidly commissioning those under construction.

(ii) Guaranteeing transnational access to all facilities for all qualified European users.

(iii) Pushing the storage-ring technology to the limit.

(iv) Paving the way to X-FELs by advanced R\&D on SASE.

In addition, Europe should closely collaborate with the USA and Russia to keep in contact with future developments concerning ERLs. 